\title{
PTEN in prefrontal cortex is essential in regulating depression-like behaviors in mice
}

\author{
Xiao-Qing Wang ${ }^{1}$, Lei Zhang $\mathbb{1}^{1,2}$, Zhong-Yuan Xia ${ }^{3}$, Jia-Yin Chen ${ }^{1,4}$, Yiru Fang $\mathbb{D}^{5,6}$ and Yu-Qiang Ding (1) ${ }^{1,4,7}$
}

\begin{abstract}
Chronic stress is an environmental risk factor for depression and causes neuronal atrophy in the prefrontal cortex (PFC) and other brain regions. It is still unclear about the molecular mechanism underlying the behavioral alterations and neuronal atrophy induced by chronic stress. We here report that phosphatase and tensin homolog deleted on chromosome ten (PTEN) is a mediator for chronic stress-induced depression-like behaviors and neuronal atrophy in mice. One-month chronic restraint stress (CRS) up-regulated PTEN signaling pathway in the PFC of mice as indicated by increasing levels of PTEN, p-MEK, and p-ERK but decreasing levels of p-AKT. Over-expression of Pten in the PFC led to an increase of depression-like behaviors, whereas genetic inactivation or knockdown of Pten in the PFC prevented the CRS-induced depression-like behaviors. In addition, systemic administration of PTEN inhibitor was also able to prevent these behaviors. Cellular examination showed that Pten over-expression or the CRS treatment resulted in PFC neuron atrophy, and this atrophy was blocked by genetic inactivation of Pten or systemic administration of PTEN inhibitor. Furthermore, possible causal link between Pten and glucocorticoids was examined. In chronic dexamethasone (Dex, a glucocorticoid agonist) treatment-induced depression model, increased PTEN levels were observed, and depression-like behaviors and PFC neuron atrophy were attenuated by the administration of PTEN inhibitor. Our results indicate that PTEN serves as a key mediator in chronic stress-induced neuron atrophy as well as depression-like behaviors, providing molecular evidence supporting the synaptic plasticity theory of depression.
\end{abstract}

\section{Introduction}

Depression is a common and devastating illness, which leads to an elevated risk for suicide ${ }^{1,2}$, as well as increased risks of cardiac disease, cerebrovascular disorders, and other medical causes of mortality ${ }^{3}$. Susceptibility to depression is influenced by a variety of genetic, epigenetic, endocrine, and environmental risk factors ${ }^{4,5}$. For instance, vulnerable individuals exposed to traumatic or stressful life events may develop depression ${ }^{6}$, and whether they develop the disease is determined by genetic make-up, exposure to prior stressful experiences, and other physiological parameters ${ }^{7}$. Studies in rodent models have

\footnotetext{
Correspondence: Lei Zhang (leizhang1120@outlook.com) or

Yu-Qiang Ding (dingyuqiang@vip.163.com)

${ }^{1}$ Key Laboratory of Arrhythmias, Ministry of Education of China, East Hospital, and Department of Anatomy and Neurobiology, Tongji University School of Medicine, Shanghai 200092, China

${ }^{2}$ Department of Anatomy, Histology and Embryology, School of Basic Medical Sciences, Fudan University, Shanghai 200032, China

Full list of author information is available at the end of the article
}

demonstrated that chronic stress causes depression-like behaviors, such as decreased sucrose intake, social defeat, and learned helplessness ${ }^{8-10}$, as well as the atrophy of neuronal processes and decrease of synapse number in the prefrontal cortex (PFC) and other brain regions ${ }^{2,11,12}$. However, it is still unclear for the molecular mechanism underlying the cellular alterations and behavioral changes induced by chronic stress.

PTEN (phosphatase and tensin homolog deleted on chromosome ten) is one of the most frequently lost or mutated tumor suppressors in human cancer, and is generally associated with advanced and metastatic disease $^{13-15}$. PTEN is a negative regulator of the phosphatidylinositol 3-kinase (PI3K)/protein kinase B (AKT)/mammalian target of rapamycin (mTOR) signaling pathway, and is also involved in the brain development, axonal regeneration, and neurodegenerative diseases ${ }^{16-19}$. PTEN deficiency in mouse brain has been shown to lead to behavioral abnormalities, 
such as altered sociability, repetitive behaviors, and anxiety $^{20,21}$. In addition, the elevated PTEN levels and lowered PI3K and Akt activities have been reported in the brain of depressed suicide victims ${ }^{22,23}$, and overexpression of PTEN causes a reduction of dendrite complexity $^{24,25}$. We thus hypothesized that PTEN may be involved in chronic stress-induced neuronal atrophy and depression-like behaviors.

A hallmark of the stress response is the activation of the hypothalamic-pituitary-adrenal (HPA) axis and increased levels of circulating glucocorticoids, providing maximum physiological support in the acute phase of the 'fight-or-flight' response ${ }^{1,26}$. Depression is associated with the hyperactivity of HPA axis ${ }^{27}$, and PTEN expression is up-regulated by the administration of glucocorticoids in A549 cells $^{28}$. Notably, chronic exposure of glucocorticoids causes the atrophy of neurons in the PFC and hippocampus $^{12,29}$, and depression-like behaviors in rodents ${ }^{30}$, raising the possibility that elevated glucocorticoids may contribute to the up-regulation of PTEN in the brain.

In this study, we found that the PTEN levels were increased in the PFC of mice treated with chronic restraint stress (CRS) or chronic administration of dexamethasone (Dex), a synthetic glucocorticoid agonist. Depression-like behaviors were also observed in mice with over-expression of Pten in the PFC, while deletion or knockdown of Pten in the PFC prevented CRS-induced depression-like behaviors. In addition, systemic administration of PTEN inhibitor reduced the CRS- or Dexinduced depression-like behaviors as well. Neuron atrophy was present in the PFC of CRS- or Dex-treated mice and was blocked by the administration of PTEN inhibitor. These data indicate that PTEN is a key factor in regulating depression-like behaviors in mice, providing a novel and promising strategy of inhibiting PTEN activity for treating this psychiatric disease.

\section{Materials and methods \\ Animals}

Eight-week-old adult male mice including wild-type C57BL/6 mice (SLAC Laboratories, Shanghai, China), Pten $^{\text {Flox/Flox }}$ mice $^{31}$ (\#006440; Jackson Laboratory, ME) and heterozygous Emx1-Cre mice ${ }^{32}$ (\#005628; Jackson Laboratory) were used. Pten ${ }^{\text {Flox/Flox }}$ mice were obtained by crossing male and female Pten ${ }^{\text {Flox/Flox }}$ mice, and heterozygous Emx1Cre mice were obtained by crossing homozygous Emx1-Cre and wild-type C57BL/6 mice. Mice were housed in groups of 5 in a cage with a 12-h light/dark cycle (lights on at 7:00 a. m.) under controlled temperature $\left(22 \pm 2{ }^{\circ} \mathrm{C}\right)$ and humidity $(50 \pm 10 \%)$, and were provided standard diet and water ad libitum. Animal care practices and all experiments were reviewed and approved by the Laboratory Animal Committee of Tongji University School of Medicine, Shanghai, China (TJmed-010-10).

\section{Intracranial viral injections}

Mice were anesthetized with sodium pentobarbital ( $1 \mathrm{~g} / \mathrm{kg}$ body weight) and $500 \mathrm{nl}$ of adeno-associated virus (AAV) (1.7 to $1.9 \times 10^{13} \mathrm{vg} / \mathrm{ml}$; Taitool Bioscience, Shanghai, China. For details, see below) was injected into the bilateral PFC using pulled glass capillary pipettes according to the atlas $(0.5 \mathrm{~mm}$ lateral to the midline, $1.54 \mathrm{~mm}$ anterior to Bregma, and $1.8 \mathrm{~mm}$ below the skull) in a double-blind way. The pipette was left in place for 5 min after each injection and then was slowly withdrawn. Silk sutures were used to close the wound after the injection. For behavioral tests, undiluted viral solution was used, while for neuron morphological analysis, a 1:2000 dilution of virus in phosphate buffer saline (PBS) was injected.

Viruses used in this study included AAV2/8-hSyn-DIOtdTomato-WPRE-bGHpA (for short, AAV2/8-DIO-tdTomato, which expressed Cre-dependent tdTomato in neurons), AAV2/8-hSyn-DIO-tdTomato-P2A-PTEN-WPREpA (AAV2/8-DIO-tdTomato-PTEN, which expressed Credependent tdTomato and PTEN separately in neurons), AAV2/9-hSyn-GFP-3Flag-WPRE-pA (AAV2/9-GFP, which expressed GFP in neurons), AAV2/9-hSyn-Cre-GFPWPRE-pA (AAV2/9-Cre, which expressed GFP-fused Cre in neurons), AAV2/9-hSyn-DIO-mCherry-miRNA (AAV2/9DIO-mCherry-miRNA, which expressed Cre-dependent mCherry and scramble miRNA separately in neurons), and AAV2/9-hSyn-DIO-mCherry-PTEN-miRNA (AAV2/9DIO-mCherry-PTEN-miRNA, which expressed Credependent mCherry and PTEN-miRNA separately in neurons).

The scramble miRNA sequence is 5'-GTCTCCA CGCGCAGTACATTT-3' and the PTEN miRNA sequence is 5'-TCGACTTAGACTTGACCTATA-3'.

\section{Chronic restraint stress (CRS)}

Mice were subjected to periodic physical restraint by immobilization in a mouse restraint apparatus $(6 \mathrm{~h} /$ day $)^{33,34}$ for 30 days, and unrestrained control mice were raised in home cage as usual. The body weight was measured every 5 days. After completion of CRS, mice were subjected to the examination of depression-like behaviors (see below) the next day.

\section{Chronic Dex treatment}

Mice were subcutaneously administered with saline or Dex $(0.2 \mathrm{mg}(10 \mathrm{ml}) / \mathrm{kg}$ body weight; HY-14648, MedChemExpress, NJ) dissolved in saline daily for 21 consecutive days in a double-blind way. The body weight was measured every 5 days, and mice were subjected for examination of depression-like behaviors after the 21-day injection of Dex.

\section{Drug administration \\ VO-Ohpic trihydrate (VO-Ohpic), an inhibitor of PTEN $^{35}(10 \mu \mathrm{g} \quad(10 \mathrm{ml}) / \mathrm{kg}$ body weight; HY-13074,}


MedChemExpress), was freshly dissolved in sterilized PBS ( $\mathrm{pH}$ 7.2), and intraperitoneally injected once per day into the mice with 30-day CRS treatment starting on day 15, or into those with 21-day Dex treatment starting on day 11, according to previous reports ${ }^{36-38}$, with modifications. The injection (VO-Ohpic or PBS) was done $1 \mathrm{~h}$ before the CRS or Dex treatment in a double-blind way, and the mice were finally subjected to the behavioral observation after the completion of 30-day CRS or 21-day Dex treatment.

\section{Hormone assays}

Mice were fasted for $8-10 \mathrm{~h}$, and blood samples were collected at 8:00 a.m. the next morning. Serum levels of corticosterone (CORT) were measured using Mouse Cortisol ELISA Kit (B163545, BIM Biosciences, CA) according to the manufacturer's protocol. The concentrations of CORT were then calculated from the appropriate standard curve and expressed as $\mathrm{ng} / \mathrm{ml}$.

\section{Quantitative real-time PCR (RT-PCR)}

PFC tissues were isolated and total RNA was extracted using Trizol reagent (RR047A, TaKaRa, Beijing, China) following the manufacturer's instructions. Three $\mu \mathrm{g}$ of total RNA was subjected to cDNA synthesis. Primers for the CRH (corticotropin-releasing hormone): 5'-CC TCAGCCGGTTCTGATCC-3' (upstream) and 5'- GCG GAAAAAGTTAGCCGCAG-3' (downstream). The primers for GAPDH: 5'- AGGTCGGTGTGAACGGATT TG-3' (upstream) and 5'- TGTAGACCATGTAGTT GAGGTCA-3' (downstream). Real-time PCR was performed using QuantiFast SYBR Green PCR Kit (204156, Qiagen, Shanghai, China), and the reaction solution consisted of $0.4 \mu \mathrm{l} / 0.4 \mu \mathrm{l}$ upstream/downstream primers, $3.2 \mu \mathrm{l}$ nuclease-free $\mathrm{H}_{2} \mathrm{O}, 1 \mu \mathrm{l}$ cDNA and $5 \mu \mathrm{l} 2 \mathrm{x}$ Mix. PCR conditions used were: $10 \mathrm{~min}$ at $95^{\circ} \mathrm{C}$ for enzyme activation followed by 40 cycles of $15 \mathrm{~s}$ denaturation at $95^{\circ} \mathrm{C}$ and $1 \mathrm{~min}$ anneal/extend at $60^{\circ} \mathrm{C}$. CRH expression levels were normalized to GAPDH with fold change differences determined using the $2^{-\Delta \Delta \mathrm{Ct}}$ method.

\section{Behavioral tests}

At the age of 12 weeks, mice were sequentially subjected to the sucrose preference test (SPT), tail suspension test (TST), forced swim test (FST), and open-field test $(\mathrm{OFT})^{39-41}$ in a double-blind way. The SPT was used to test anhedonia-like behavior. Briefly, mice were given a free choice between two bottles of the same color: one with $0.1 \%$ sucrose solution and the other with water. It was noteworthy that the position of the bottles was changed after $12 \mathrm{~h}$. At the end of $24 \mathrm{~h}$, liquid consumption was measured, and the sucrose preference was calculated as the ratio of the volume of $0.1 \%$ sucrose solution consumed to the total liquid intake. In the TST and FST, the immobility time was recorded in the last $4 \mathrm{~min}$.
Locomotor activity was assessed in open field $(40 \mathrm{~cm} \times$ $40 \mathrm{~cm}$ ) and total distance was measured in $30 \mathrm{~min}$ sessions for each animal.

\section{Analysis of neuronal morphology}

Brain slices (35- $\mu \mathrm{m}$ thick) were collected 30 or 21 days after intracranial viral injections. Confocal images of individual pyramidal neurons in layers II/III of PFC were captured with $40 \mathrm{X}$ oil objective lens at the resolution of $1024 \times 1024$ pixels by Nikon A1R laser-scanning confocal microscope. The cell soma size was measured by outlining the soma and calculation of area in squared micrometers. For Sholl analysis, concentric circles with $10 \mu \mathrm{m}$ differences in radius were drawn around the cell soma, and the number of dendrites intercrossing each circle was counted. The neuronal dendrites were traced and total dendritic length was calculated ${ }^{25,42}$. All the morphological analysis was done in a double-blind way.

\section{Immunofluorescence}

Brain slices (35- $\mu \mathrm{m}$ thick) were pretreated with sodium citrate $(0.05 \mathrm{M}, \mathrm{pH} 6.0)$ for $5 \mathrm{~min}$ at $95^{\circ} \mathrm{C}$ for antigen retrieval and this procedure might largely diminish the GFP or tdTomato fluorescence. Then these slices were incubated with rabbit anti-PTEN antibody (1:1000; ab32199, Abcam, Shanghai, China), anti-p-AKT antibody (1:1000; \#4060, CST, MA), or goat anti-GFP antibody (1:2000; NB100-1770, Novus Biologicals, CO) at $4{ }^{\circ} \mathrm{C}$ overnight, and incubated with biotinylated horse antirabbit IgG (1:500; Jackson ImmunoResearch, PA) or Alexa Fluor 488 donkey anti-goat IgG (1:500; Invitrogen, Shanghai, China) at room temperature for $3 \mathrm{~h}$, followed by incubation with streptavidin-Cy3 or streptavidin-Cy5 (1:1000; Jackson ImmunoResearch) and counterstaining with Hoechst 33258 (1:2000, Sigma, Shanghai, China) at room temperature for $10 \mathrm{~min}$. Images were captured with a Nikon A1R laser-scanning confocal microscope.

\section{Western blots}

The PFC tissue was dissected out and lysed with cold RIPA lysis buffer (\#89901, Invitrogen). The collected protein was diluted in sample buffer (LT101s, EpiZyme, MA) and boiled for $10 \mathrm{~min}$. Twenty $\mu \mathrm{g}$ of protein was loaded onto 10\% SDS-PAGE gel and transferred to nitrocellulose membranes. Antibodies used for Western blot were rabbit anti-PTEN (1:1000; ab32199, Abcam), rabbit anti-p-PTEN (1:1000; \#9551, Abcam), mouse anti-p-ERK1/2 (1:1000; \#4370, CST), mouse anti-ERK1/2 (1:1000; \#4696, CST), rabbit anti-p-MEK1 (1:1000; \#26975, CST), rabbit antiMEK1 (1:1000; \#12671, CST), rabbit anti-AKT (1:1000; \#4691, CST), rabbit anti-p-AKT(1:1000; \#4060, CST), and rabbit anti-GAPDH (1:2000; LF206, EpiZyme). Protein bands were detected using enhanced chemiluminescence reagents (\#1525703, Millipore, Hong Kong, China). 


\section{Statistical analysis}

All data were tested for normal distribution and statistical analysis was then carried out using two-tailed Student's $t$ test and one-way or two-way ANOVA with post hoc Tukey's test (GraphPad Prism, v8.0). Data are presented as mean \pm SEM. $p$ values less than 0.05 were considered significant. All experiments were replicated for at least 3 times.

\section{Results}

\section{CRS treatment up-regulates PTEN levels in the PFC}

CRS is widely used in inducing depression-like behaviors in rodents ${ }^{43,44}$. To validate our CRS protocol, the behavior tests including the SPT, TST, FST, and OFT were performed (Fig. 1a). As expected, CRS treatment resulted in a decrease of sucrose preference and an increase of immobility time in both TST and FST (Fig. 1b-d). Because the hyperactivity of the HPA axis is involved in the pathogenesis of depression ${ }^{45,46}$, CRStreated mice exhibited increased CORT levels in the serum, and increased CRH transcription in the PFC (Fig. 1e, f), consistent with the previous results ${ }^{47}$. The locomotor activity was not changed in CRS-treated mice as shown by similar total traveled distance relative to controls (Fig. 1g). Finally, there was a gradual increase of body weight in control mice during the 30-day period, but this was not present in CRS-treated mice (Fig. 1h). Taken together, our CRS treatment induced the appearance of depression-like behaviors in mice.

To explore if PTEN is involved in CRS-induced depression, we examined the expression levels of PTEN and its associated molecules in the PFC (Fig. 2a), which is a critical brain region for depression ${ }^{48,49}$. Western blots showed that the levels of PTEN were significantly increased in CRS-treated mice (Fig. 2b, c). The components of PTEN signaling pathway showed corresponding changes as reflected by the increased phosphorylation of ERK1/2 and MEK1, and decreased phosphorylation of AKT in CRS-treated mice (p-ERK1/2/ERK1/2, p-MEK1/ MEK1, and p-AKT/AKT ratio; Fig. 2b, d-f). Note that the levels of PTEN phosphorylation, AKT, ERK1/2 and MEK1 were not changed (Fig. 2b-f). Thus, our data demonstrate that PTEN signaling pathway is up-regulated in CRStreated mice, suggesting possible involvement of PTEN in the development of depression-like behaviors in mice.
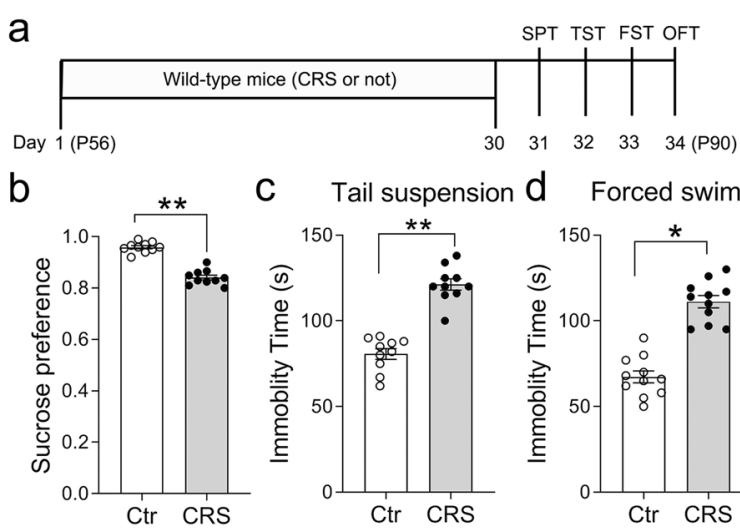

C Tail suspension d Forced swim
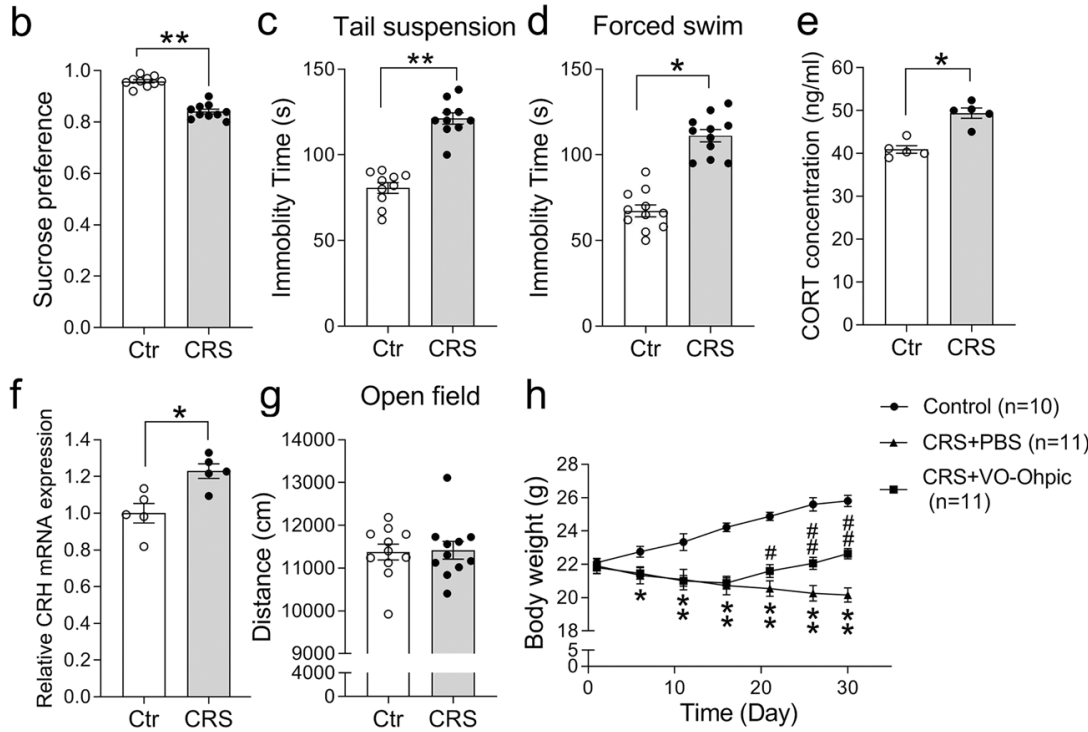

Fig. 1 CRS treatment induces depression-like behaviors and elevates HPA activity. a Diagram of experiment design and timeline. $\mathbf{b}-\mathbf{d}$ The CRS treatment resulted in decreased sucrose preference (b) and increased immobility time in the TST (c), and FST (d). $n=10$ for each group in sucrose preference test and TST, $n=11$ for each group in FST. e, $\mathbf{f}$ The CRS treatment significantly increased the serum levels of CORT (e) and transcription levels of CRH in the PFC (f). $\mathbf{g}$ There were no significant changes in the total traveled distance in the OFT. $n=11$ for each group. $\mathbf{h}$ A significant difference was observed in the body weight between CRS-treated mice and control mice during the 30-day period, but the reduction of body weight in CRS-treated mice could be partially reversed when PTEN inhibitor VO-Ohpic was intraperitoneally injected once per day from day 15 . All the data are presented as mean \pm SEM. Data were analyzed using Student's $t$-tests $(\mathbf{b}-\mathbf{g})$ and two-way repeated measures ANOVA (h). ${ }^{*} p<0.05,{ }^{* *} p$ $<0.01$ (control mice versus CRS-treated mice); ${ }^{\#} p<0.05,{ }^{\# \#} p<0.01$ (CRS-treated mice versus CRS-treated mice with VO-Ohpic). CRS, chronic restraint stress; FST, forced swim test; OFT, open-field test; SPT, sucrose preference test; TST, tail suspension test; VO-Ohpic, VO-Ohpic trihydrate. 


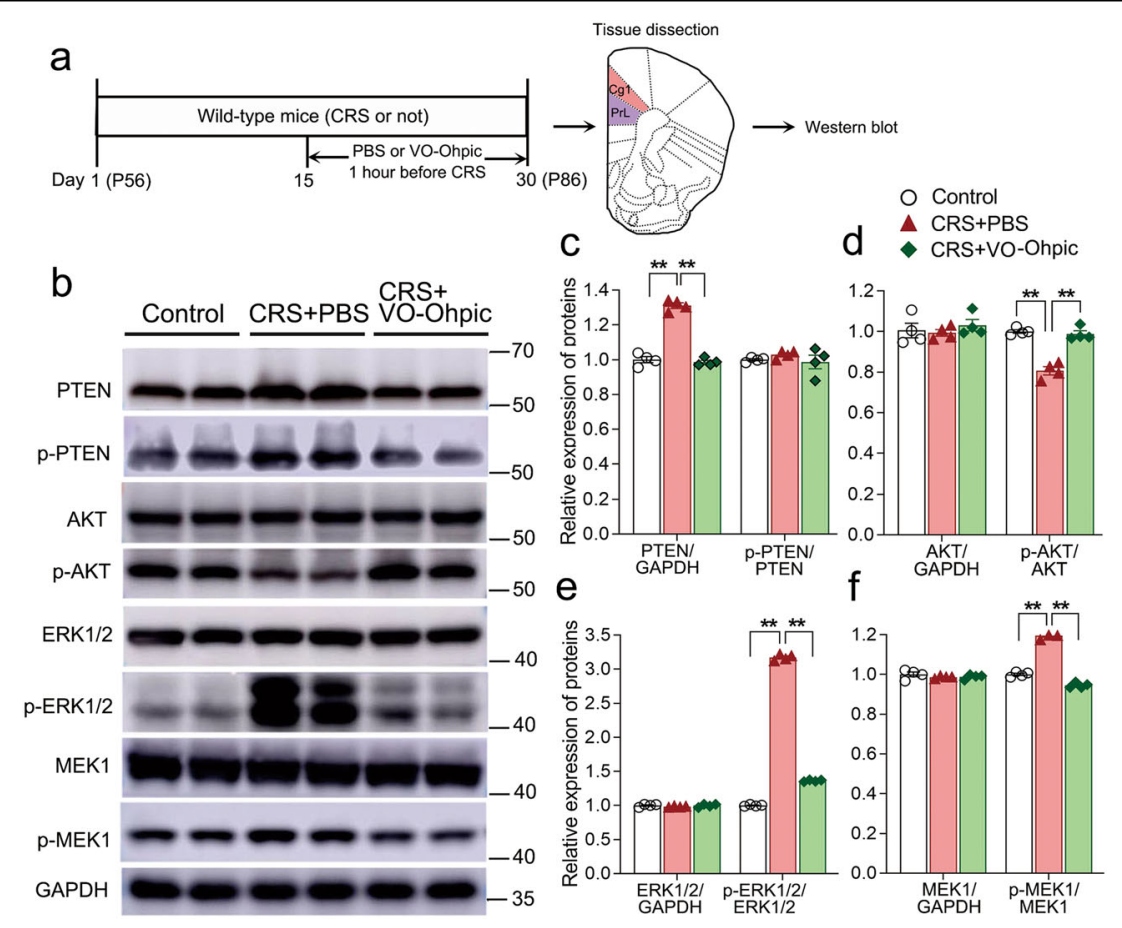

Fig. 2 The activity of PTEN signaling pathway is up-regulated in the PFC of CRS-treated mice, and it can be prevented by the administration of PTEN inhibitor. a Diagram of experiment design and timeline. Cortical tissues from the two subregions of PFC, Cg1, and PrL were collected for Western blots. b-f One-month CRS treatment led to the increase in the levels of PTEN, p-MEK1, and p-ERK1/2 and decrease of p-AKT in the PFC, but these changes were not observed in the CRS-treated mice receiving daily injection of PTEN inhibitor during the period of days 15-30. Note that the levels of p-PTEN, ERK1/2, AKT, and MEK1 were not changed by the CRS-treatment or application of PTEN inhibitor. $n=4$ in each group and data are presented as mean \pm SEM. Two-way ANOVA with post hoc Tukey's test, ${ }^{*} p<0.05$, ${ }^{* *} p<0.01$. Cg1, cingulate cortex, area 1; CRS, chronic restraint stress; PrL, prelimbic cortex; VO-Ohpic, VO-Ohpic trihydrate.

PTEN in the PFC is critical for depression-like behaviors in mice

To explore possible effects of increased PTEN in the PFC on depression-like behaviors, we injected Credependent PTEN-overexpressing AAV (AAV2/8-DIOtdTomato-PTEN) or control virus (AAV2/8-DIO-tdTomato) into the PFC of Emx1-Cre mice (Fig. 3a). Injection of AAV2/8-DIO-tdTomato-PTEN resulted in an increase of PTEN levels in Emx1-Cre mice compared to those with injection of control AAV2/8-DIO-tdTomato (Supplementary Fig. 1a). Thirty days later, we performed behavioral examination of Emx1-Cre mice with the injection of virus. Results showed that the sucrose preference was significantly reduced (Fig. 3b) and the immobility time in the TST and FST was obviously increased (Fig. 3c, d) in the mice with injection of AAV2/8-DIO-tdTomato-PTEN compared to those with injection of control AAV2/8DIO-tdTomato. Note that the total traveled distance in the OFT was not different between the groups (Fig. 3e), while the entries to the center region were decreased in AAV2/8-DIO-tdTomato-PTEN-injected Emx1-Cre mice (Supplementary Fig. 2). Similarly, we performed overexpression of PTEN by co-injection of AAV2/9-Cre with
AAV2/8-DIO-tdTomato-PTEN into the PFC of wild-type mice (Fig. 3f). There was a marked increase in PTEN immunoreactivity in the cortical neurons co-infected with AAV2/9-Cre and AAV2/8-DIO-tdTomato-PTEN compared to those with AAV2/9-Cre and AAV2/8-DIOtdTomato (Supplementary Fig. 3). Behavioral examination was performed 30 days after the injection and similar alterations were obtained as mentioned above (Fig. $3 g-j$ ). Thus, depression-like behaviors are increased in mice with over-expression of PTEN in the PFC.

Next, we set out to explore if inactivation of Pten in the PFC affects depression-like behaviors. In the first set of experiments, we injected AAV2/9-Cre into the PFC of Pten ${ }^{\text {Flox/Flox }}$ mice to observe how depression-like behaviors are changed in these mice with or without one-month CRS treatment (Fig. 3k). Western blots showed a significant reduction of PTEN levels in the PFC of Pten ${ }^{\text {Flox/Flox }}$ mice with injection of AAV2/9-Cre compared to those with injection of control AAV2/9-GFP (Supplementary Fig. 1b). At cellular level, the deletion of Pten in Cre-expressing neurons was confirmed by immunostaining of PTEN and the presence of intense p-AKT immunoreactivity (Supplementary Fig. 4). Behavioral examination showed that there 


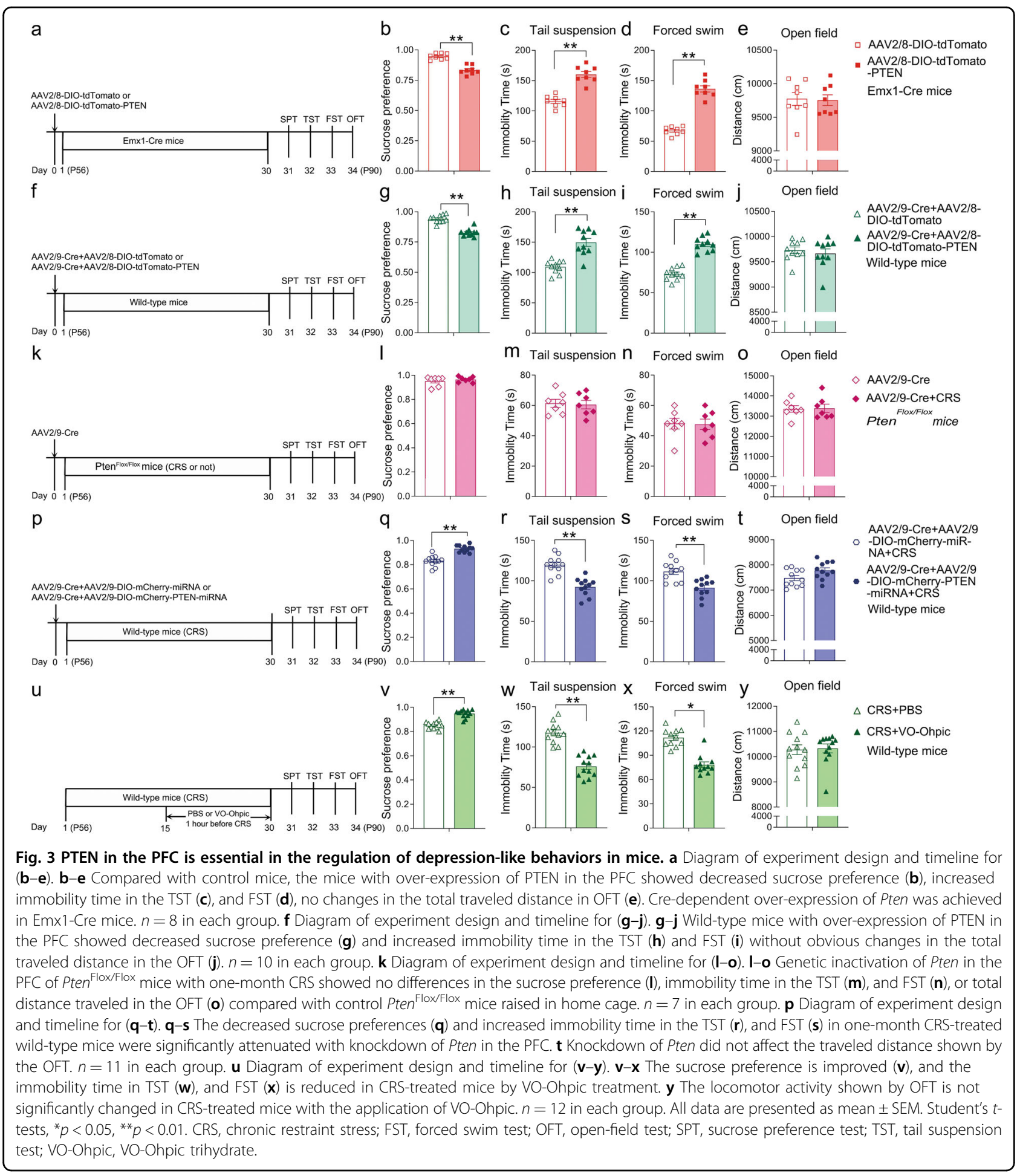

were no differences in the sucrose preference (Fig. 3l), the immobility time in the TST and FST (Fig. 3m, n), or the total distance traveled in the OFT (Fig. 3o) between the CRStreated and non-CRS-treated Pten ${ }^{\text {Flox/Flox }}$ mice with injection of AAV2/9-Cre. Thus, the CRS-induced depression-like behaviors are no longer present in the mice with genetic inactivation of Pten in the PFC.

To further confirm this, we performed another set of experiments in wild-type mice with knockdown of Pten in the PFC. A mix of Cre-dependent miRNA against Pten 


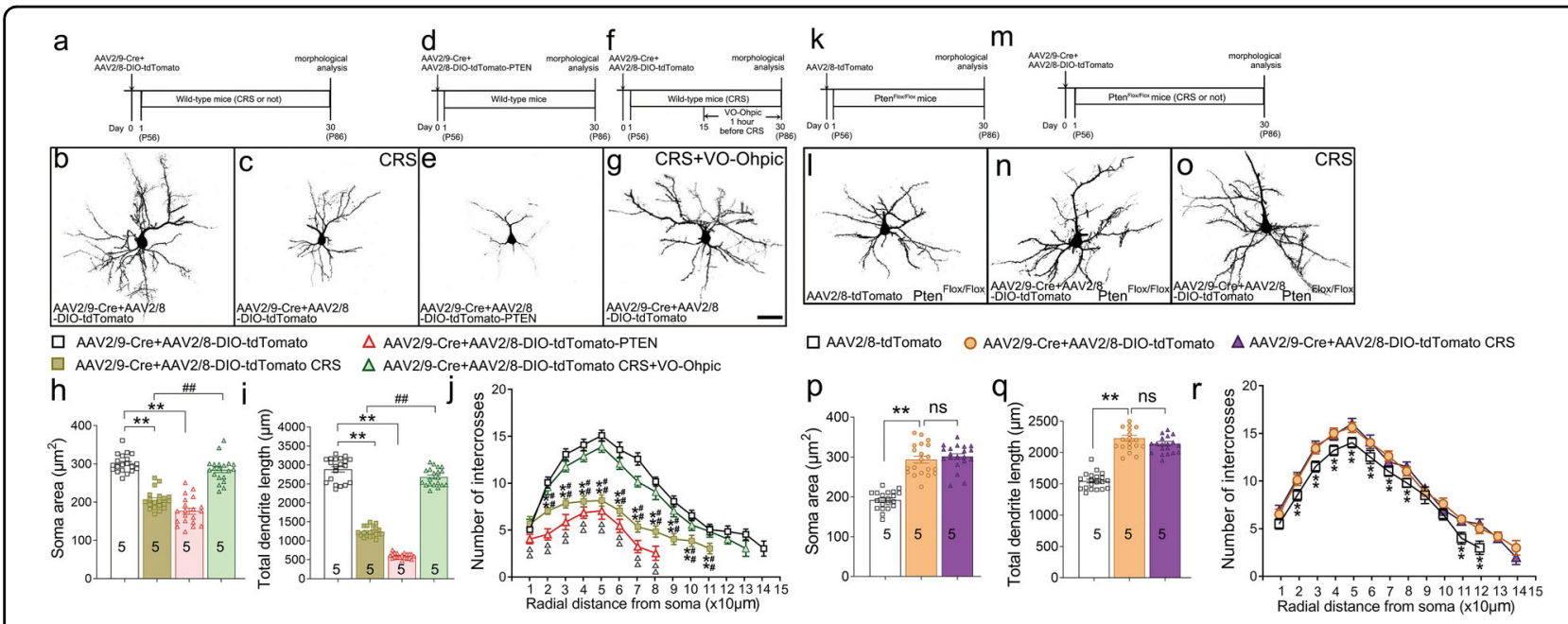

Fig. 4 PTEN is a key factor involved in the CRS-induced dendrite atrophy. a Diagram of experiment design and timeline for (b, c). b, c Representative images showing the dendrite tree of pyramidal neurons in layers I//III of PFC from control mice and CRS-treated mice. $\mathbf{d}$ Diagram of experiment design and timeline for (e). e Representative image showing the dendrite tree of pyramidal neurons in layers II/III of PFC from mice with over-expression of Pten. $\mathbf{f}$ Diagram of experiment design and timeline for $(\mathbf{g})$. $\mathbf{g}$ Representative image showing the dendrite tree of pyramidal neurons in layers II/III of PFC from CRS-treated mice with VO-Ohpic administration. $\mathbf{h}-\mathbf{j}$ Statistical data showing the soma size (h), total length of dendrites (i), and dendritic branching (j) of the PFC neurons in indicated groups. $n=20$ cells from 5 mice in each group and all data are presented as mean \pm SEM. One-way ANOVA (h, i), ${ }^{* *} p<0.01$ versus controls, ${ }^{\# \#} p<0.01$ (CRS-treated mice versus CRS-treated mice with VO-Ohpic). Two-way ANOVA with post hoc Tukey's test $\mathbf{j}$ ), ${ }^{*} p<0.05,{ }^{* *} p<0.01$ (control mice versus CRS-treated mice); ${ }^{\#} p<0.05,{ }^{\# \#} p<0.01$ (CRS-treated mice versus CRStreated mice with VO-Ohpic); ${ }^{\Delta} p<0.05 ;{ }^{\Delta \Delta} p<0.01$ (control mice versus mice with over-expression of Pten). $\mathbf{k}$ Diagram of experiment design and timeline for (I). I Representative image showing the dendrite tree of pyramidal neuron in layers II/III of PFC from control mice. $\mathbf{m}$ Diagram of experiment design and timeline for $(\mathbf{n}, \mathbf{o})$. $\mathbf{n}$, o Representative images showing the dendrite tree of pyramidal neuron in layers II/III of PFC from Ptendeficient neurons without or with CRS, respectively. Deletion of Pten was achieved by the injection of AAV2/9-Cre in Pten ${ }^{\text {Flox/Flox }}$ mice. $\mathbf{p}$-r $\mathbf{r}$ Statistical data showing the soma size $(\mathbf{p})$, total length of dendrites $(\mathbf{q})$, and dendritic branching $(\mathbf{r})$ of the PFC neurons in indicated groups. $n=20$ cells from 5 mice in each group and all data are presented as mean \pm SEM. One-way ANOVA $(\mathbf{p}, \mathbf{q}),{ }^{* *} p<0.01$ versus controls. Two-way ANOVA with post hoc Tukey's test $(\mathbf{r}),{ }^{*} p<0.05,{ }^{* *} p<0.01$ (control neuron versus Pten-deficient neurons with CRS). Scale bar $=40 \mu \mathrm{m}(\mathbf{b}, \mathbf{c}, \mathbf{e}, \mathbf{g}, \mathbf{I}, \mathbf{n}$, and $\mathbf{o})$. CRS, chronic restraint stress; VO-Ohpic, VO-Ohpic trihydrate.

expression virus (AAV2/9-DIO-mCherry-PTEN-miRNA) and AAV2/9-Cre was injected into the PFC of wild-type mice (Fig. 3p), and the knockdown efficacy was assessed by Western blot (Supplementary Fig. 5). One day after the virus injection, all mice were subjected to the CRS, and behavioral observation was done 30 days later (Fig. 3p). In comparison with control mice with injection of both AAV2/9-Cre and AAV2/9-DIO-mCherry-miRNA, those with injection of AAV2/9-Cre and AAV2/9-DIOmCherry-PTEN-miRNA exhibited improved sucrose preferences (Fig. 3q) and decreased immobility time in the TST and FST (Fig. 3r, s) with no obvious differences in the total distance traveled in the OFT (Fig. 3t). Taken together, our data obtained by the manipulation of PTEN levels in the PFC demonstrate that PTEN is essential in the regulation of depression-like behaviors in mice.

\section{PTEN is implicated in CRS-induced atrophy of cortical neurons in mice}

It has been reported that chronic stress leads to the reduction of dendritic length and spine density of cortical neurons ${ }^{1,2,12}$. Considering the role of PTEN in regulating the dendrite morphology ${ }^{24}$, we speculated that PTEN is involved in the CRS-induced dendrite atrophy which may be one of the cellular mechanisms underlying the onset of depressionlike behaviors. To this end, we first examined the dendrite morphology of cortical pyramidal neuron in the layers II/III of PFC of one-month CRS-treated wild-type mice by coinjection of AAV2/9-Cre and AAV2/8-DIO-tdTomato one day before the commencement of the CRS treatment (Fig. 4a); tdTomato was used to trace the dendrite morphology of infected neurons. Consistent with previous reports $^{50,51}$, the atrophy of cortical neurons was evident in the CRS-treated mice (Fig. 4b, c), as shown by significant reductions in the soma size (Fig. 4h), the total length of dendrites (Fig. 4i), and number of dendrite branches (Fig. 4j) relative to those of control mice. Next, we moved to examine how the dendrite tree was changed after the over-expression of Pten in the PFC. As mentioned above, the AAV2/9-CreGFP and AAV2/8-DIO-tdTomato-PTEN virus was coinjected into the PFC of wild-type mice (Fig. 4d), and tdTomato could depict the dendrite morphology of infected neurons (Fig. 4e). Importantly, the cortical neurons with over-expression of PTEN showed simplified dendrites as indicated by a significant reduction in the total length of dendrites and number of dendrite branches as compared 
with those of control mice with co-injection of AAV2/9-Cre and AAV2/8-DIO-tdTomato virus (Fig. 4h-j). Thus, the CRS treatment or over-expression of Pten leads to the dendrite atrophy of cortical neurons in the PFC.

We then moved to examine the dendrite morphology when Pten is inactivated. As described above, a mix of AAV2/9-Cre and AAV2/8-DIO-tdTomato (Fig. 4m) or AAV2/8-tdTomato alone (Fig. 4k) was injected into the PFC of $P t e n^{\text {Flox/Flox }}$ mice. One day after the injection, the mice receiving AAV2/9-Cre and AAV2/8-DIO-tdTomato were randomly divided into two groups: one is raised in home cage for one month, and the other is subjected to one-month CRS treatment (Fig. 4m). Deletion of Pten was confirmed by the loss of PTEN immunoreactivity (Supplementary Fig. $4 \mathrm{a}-\mathrm{c}$ ) and increased p-AKT immunoreactivity (Supplementary Fig. $4 \mathrm{~d}-\mathrm{f}$ ) in tdTomato-positive neurons compared with adjacent uninfected neurons. Sholl analysis showed that the cortical neurons lacking Pten had larger soma size (Fig. 4l, n, p) and increased dendrite length (Fig. 4l, n, q) and branches (Fig. 4l, n, r) compared with control mice. Critically, these alterations of Pten-deficient neurons were not observed in the mice with one-month CRS treatment (Fig. 4o-r). These results suggest that the inactivation of Pten in PFC neurons endows them with the ability against the CRS-induced neuronal atrophy, which may serve as one of the cellular mechanisms underlying the essential role of PTEN in regulating depression-like behaviors in mice.

\section{PTEN inhibitor VO-Ohpic prevents the onset of CRS- induced depression-like behaviors in mice}

To provide more evidence supporting the critical role of PTEN in regulating depression-like behaviors, we applied VO-Ohpic, an inhibitor of PTEN $^{35}$ by intraperitoneal injection in wild-type mice. VO-Ohpic was applied in one-month CRS-treated mice starting on day 15 until the end of CRS treatment (Figs. 2a, 3u, and 4f). Western blots showed that the 2-week application of VO-Ohpic indeed increased the levels of p-AKT with unchanged total AKT compared to those with CRS treatment only (Fig. 2b, d). In addition, the level of PTEN and phosphorylation of ERK1/2 and MEK1 were reduced in the CRS-treated mice with the injection of VO-Ohpic compared with CRStreated mice without VO-Ohpic treatment (Fig. 2b, c, e, f), showing that the PTEN activity is suppressed by the application of VO-Ohpic. Behavioral observations (Fig. $3 \mathrm{u}$ ) revealed that the sucrose preference is improved (Fig. 3v), and immobility time in both TST and FST was reduced compared with controls (Fig. $3 \mathrm{w}, \mathrm{x}$ ). In addition, VO-Ohpic treatment prevented the decrease of body weight in CRS-treated mice (Fig. 1h) without affecting locomotor activity shown by OFT (Fig. 3y). Lastly, inhibition of PTEN with VO-Ohpic prevented the atrophy induced by CRS (Fig. 4g-j). Thus, systemic administration of VO-Ohpic is able to prevent the development of CRSinduced depression-like behaviors in mice.

\section{Elevated glucocorticoids are involved in PTEN-regulated depression-like behaviors in mice}

Our results showed the increase of serum CORT levels and CRH transcriptions in the PFC of the CRS-treated mice, and increased activity of HPA is believed to be one of the mechanisms underlying the development of depression ${ }^{52}$. It is likely that the elevated glucocorticoids contribute to the increased PTEN activity and the depression-like behaviors in the CRS-treated mice. Dex is a synthetic glucocorticoid agonist and chronic Dex treatment is widely used for inducing depression-like behaviors in rodents ${ }^{53-55}$, and thus it was used to explore: (i) how the PTEN levels are changed in Dex-treated mice, and (ii) whether suppressing PTEN activity is able to attenuate the depression-like behaviors in Dex-treated mice.

To ensure the validity of the chronic Dex model, the behavior tests including the SPT, TST, and FST were performed (Fig. 5a). As expected, 21-day Dex treatment prevented the increase of body weight (Fig. 5b), and resulted in a decrease of sucrose preference (Fig. 5c) and increase of immobility time in both TST and FST (Fig. 5d, e). Like the CRS-treated mice, serum CORT levels and CRH transcription in the PFC were increased (Fig. 5f, g). Thus, our Dex protocol indeed induced depression-like behaviors in mice. To explore if PTEN and its associated proteins were involved in the Dex-induced depression, we examined the expression levels of PTEN, AKT, p-AKT, ERK1/2, p-ERK1/2, MEK1, and p-MEK1 in the PFC (Fig. 5h). Western blots showed that the levels of PTEN and the phosphorylation of ERK1/2 and MEK1 were significantly increased (Fig. 5i, j, l, m), while the phosphorylation of AKT was decreased in Dex-treated mice (Fig. 5i, k). Thus, our data demonstrate that PTEN activity is upregulated in the PFC of Dex-treated mice.

Then, the PTEN inhibitor was administrated daily starting on day 11 till the end of Dex treatment on day 21 (Fig. 5a, h). The behavior tests showed that the VO-Ohpic treatment prevented the decrease of body weight (Fig. 5b), improved the sucrose preference, and reduced the immobility time in the TST and FST (Fig. 5c-e). Changes in the levels of PTEN, p-AKT, p-ERK1/2, and p-MEK1 were reversed by VO-Ohpic in Dex-treated mice (Fig. 5i-m). Next, the dendrite morphology of PFC neurons was examined in the same way (Fig. 5n). As reported previously $^{56}$, the 21-day Dex treatment led to PFC neuron atrophy (Fig. 5o, p), revealed by significant reduction of soma size (Fig. 5r), total dendritic length (Fig. 5s), and number of branches (Fig. 5t) relative to those of controls, and these alterations no longer existed after the administration of PTEN inhibitor (Fig. 5o-t). Our data suggest that that glucocorticoids may serve as a mediator in the 


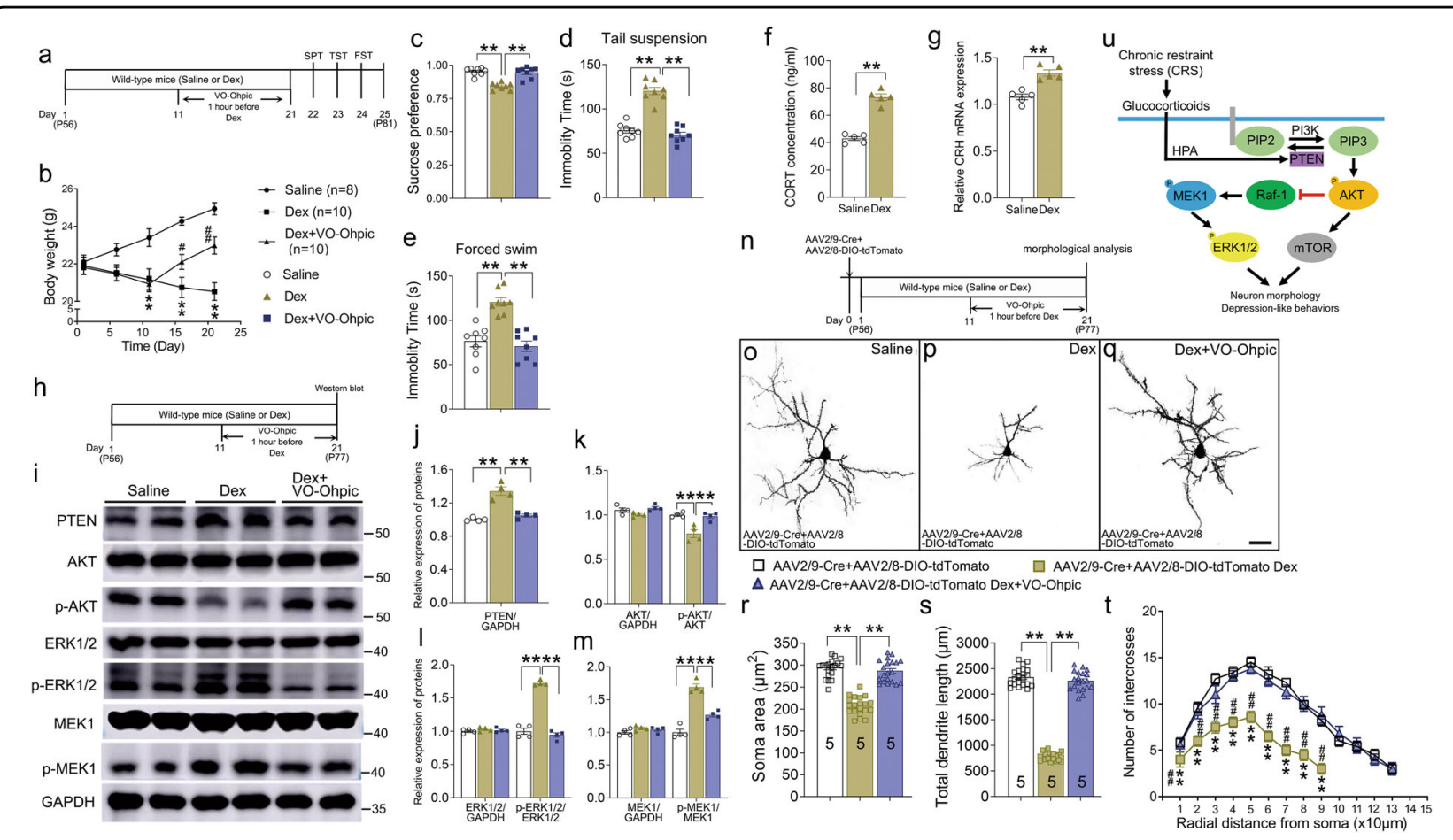

Fig. 5 PTEN inhibitor VO-Ohpic prevents depression-like behaviors and neuron atrophy in Dex-treated mice. a Diagram of experiment design and timeline for (b-e). b A significant reduction in body weight was observed after 21-day Dex treatment, but it could be partially reversed by intraperitoneal injection of VO-Ohpic into the mice starting on day 11 . Data were analyzed using two-way repeated measures ANOVA, ${ }^{*} p<0.05,{ }^{* *} p$ $<0.01$ (Saline versus Dex-treated mice); ${ }^{\#} p<0.05, \# p<0.01$ (Dex-treated mice versus Dex-treated mice with VO-Ohpic). c-e Dex resulted in decreased sucrose preference $(\mathbf{c})$, and increased immobility time in TST (d) and FST (e). $n=8$ in each group and data were analyzed using one-way ANOVA, ${ }^{* *} p$ $<0.01 . \mathbf{f}, \mathbf{g}$ The levels of CORT in the serum and CRH transcription in the PFC were increased in Dex-treated mice. Data were analyzed using Student's $t$-tests, ${ }^{* *} p<0.01$. $\mathbf{h}$ Diagram of experiment design and timeline for $(\mathbf{i}-\mathbf{m}) . \mathbf{i}-\mathbf{m}$ Three-week Dex treatment led to increased levels of PTEN, $p-M E K 1$, and $\mathrm{p}$-ERK1/2 and reduced level of $\mathrm{p}-\mathrm{AKT}$, and these changes were reversed by VO-Ohpic starting on day 11 . Note that the levels of ERK1/2, AKT, and MEK1 were not changed by the Dex treatment or application of PTEN inhibitor. $n=4$ in each group and data are presented as mean \pm SEM. One-way ANOVA $(\mathbf{j}),{ }^{*} p<0.05,{ }^{* *} p<0.01$. Two-way ANOVA with post hoc Tukey's test $(\mathbf{k}-\mathbf{m}),{ }^{*} p<0.05,{ }^{* *} p<0.01$. $\mathbf{n}$ Diagram of experiment design and timeline for $(\mathbf{o}-\mathbf{q})$. $\mathbf{0}-\mathbf{q}$ Representative images of pyramidal neuron in layers II/III of PFC neurons of control mice and Dex-treated mice with or without VOOhpic administration, respectively. Scale bar $=40 \mu \mathrm{m}$. $\mathbf{r}$-t Statistical data showing the soma size $(\mathbf{r})$, total length of dendrites $(\mathbf{s})$, and dendritic branching (t) of the PFC neurons in indicated groups. $n=20$ cells from 5 mice in each group, and all data are presented as mean \pm SEM. One-way ANOVA $(\mathbf{r}, \mathbf{s}),{ }^{* *} p<0.01$. Two-way ANOVA with post hoc Tukey's test $(\mathbf{t}),{ }^{*} p<0.05,{ }^{* *} p<0.01$ (control mice versus Dex-treated mice); ${ }^{\#} p<0.05$, ${ }^{\# \#} p<$ 0.01 (Dex-treated mice versus Dex-treated mice with VO-Ohpic). u Model of PTEN signaling pathway in CRS-induced neuronal atrophy and depression-like behaviors. CORT, corticosterone; CRH, corticotropin-releasing hormone; CRS, chronic restraint stress; Dex, dexamethasone; FST, forced swim test; HPA, hypothalamic-pituitary-adrenal axis; OFT, open-field test; SPT, sucrose preference test; TST, tail suspension test; VO-Ohpic, VO-Ohpic trihydrate.

CRS-induced up-regulation of PTEN and subsequently depression-like behaviors in mice.

\section{Discussion}

In this study, we addressed the role of PTEN in stressinduced depression-like behaviors in mice. First, CRS and Dex treatments up-regulates PTEN levels in the PFC. Second, over-expressing Pten in the PFC results in deleterious effects on dendrite tree and the increase of depression-like behaviors, whereas deletion or knockdown of Pten is able to prevent CRS-induced depression-like behaviors and neuronal atrophy in the PFC. Thus, a model of PTEN signaling pathway in neuronal morphology and depression-like behaviors is proposed
(Fig. 5u). Although the FST is also considered as a measurement for stress response, not directly related to depression $^{57}$, PTEN over-expression in the PFC increased such stress (Fig. 3d, i), which would promote the development of depression. Potential application in treating depression is shown by systemic administration of PTEN inhibitor based on the behavioral and neuronal morphological data. In addition, our data are essentially consistent with another study concerning PTEN in 5-HT neurons in dorsal raphe nucleus $(D R N)^{38}:(1)$ PTEN is elevated in 5-HT neurons by CRS; (2) depression-like behaviors are reduced in Pten cKO mice; and (3) dendritic complexity is increased in Pten-deficient 5-HT neurons. Together, these results suggest that PTEN in 
PFC-DRN network may be critical in regulating depression-like behaviors.

A wealth of evidence indicates that the PFC is a key component of the corticostriatal circuits which are thought to generate pathological emotional behavior and accompanied physiological disturbance ${ }^{58,59}$. Glucocorticoids regulate the termination of the stress response through negative feedback at the level of the hypothalamus and pituitary, as well as other brain regions such as the PFC, hippocampus, and amygdala $^{60}$. A well-documented consequence of chronic stress exposure is impaired negative feedback of the HPA axis with increased levels of corticosteroids and $\mathrm{CRH}^{61-63}$, which is observed in the majority of depressed patients ${ }^{64}$ and CRS-treated mice as shown in the present study. The previous studies have observed an increase in PTEN protein levels in the PFC of depressed suicide victims $^{23}$. What could be the remote cause of the PTEN dysregulation in the PFC of depressive brain? It has been reported that PTEN expression is up-regulated by the administration of glucocorticoids in A549 cells $^{28}$. Our data indicate that chronic Dex treatment up-regulates PTEN levels in the PFC, and the application of PTEN inhibitor prevents the onset of Dex-induced depression-like behaviors in mice. On the basis of these findings, it is likely that glucocorticoids serve as a mediator in the stress-induced upregulation of PTEN and depression-like behaviors in mice.

PTEN mutations were associated with autism spectrum disorder (ASD) $)^{65,66}$ and found in about $2 \%$ of total ASD patients ${ }^{67}$. A current model suggests PTEN mutationinduced increase of dendritic branching in PFC may disrupt the brain connectivity and then cause ASD-related neurobehavioral deficits ${ }^{68}$. We showed that knockout of PTEN in individual neurons led to hypertrophy (Fig. 4l, $\mathrm{n}-\mathrm{r}$ ) and over-expression of PTEN resulted in neuronal atrophy (Fig. 4c, e, h-j), which supports this model. PTEN is widely expressed in the brain and is present at both preand post-synaptic sites ${ }^{69,70}$. The PTEN/PI3K pathway is essential for some important aspects of synaptic function and plasticity. Interestingly, the blockade of Akt-mTOR signaling completely disrupted ketamine-induced synaptogenesis and behavioral responses in animal models of depression $^{71-75}$. Inactivation of PTEN in the differentiated neurons of the cerebral cortex resulted in increased response to sensory stimuli with neuronal hypertrophy, including hypertrophic and ectopic dendrites and axon tracts with increased synapses in mice ${ }^{76}$. In contrast, excess of PTEN restricts neuronal growth, leading to a substantial decrease in dendritic branching and synapse number ${ }^{77}$. In addition, the hippocampal granule neurons or dorsal raphe 5-HT neurons with PTEN deletion receive more synaptic inputs ${ }^{38,78-80}$ and show increased excitability in adult mice ${ }^{78-80}$, and further studies are needed to examine if similar alterations occur in Pten-deficient pyramidal neurons in the PFC.
According to the synaptic theory of depression, extrinsic factors such as chronic stress leads to impairments of synaptic plasticity at both morphological and functional levels, and traditional antidepressants (e.g., selective serotonin reuptake inhibitors) and new category of antidepressants (e.g., ketamine) are able to restore the synaptic plasticity via multiple intracellular pathways including AKT-mTOR ${ }^{1,2,81}$. Our results demonstrated essential roles of PTEN, negative regulator of AKT/ mTOR signaling, in regulating depression-like behaviors as well as CRS- and Dex-induced neuronal atrophy in mice. Thus, exploring potential chemicals which negatively regulate AKT/mTOR signaling may be helpful for developing new antidepressant. In addition to influencing the AKT-mTOR signaling pathway, PTEN was identified to be localized in the nucleus ${ }^{82,83}$ and regulate genomic stability and global gene expression ${ }^{84,85}$. Thus, deletion of PTEN is also likely to lead to chromatin decondensation and transcription activation of genes for cell growth and mitigation of depression-like behaviors.

Our results suggest that inhibiting PTEN activity can be of therapeutic benefit in treating depression. VO-Ohpic used in this study is a specific vanadium-based PTEN inhibitor ${ }^{86}$. It is able to antagonize the effect of PTEN over-expression, enhance the activity of PI3K/AKT signaling pathway, and alleviate myocardial cell apoptosis ${ }^{87}$, and it is also able to inhibit the expression of pro-inflammatory cytokines IL-1 $\beta$ and TNF- $\alpha$ and up-regulate anti-inflammatory IL-10 expression in a sudden cardiac arrest model ${ }^{88}$. On the other hand, the initial consideration of developing a PTEN inhibitor in potential clinical application is discouraged by concerns that long-term systemic PTEN inhibition may lead to increased cancer risk, and because of evidence that even modest reductions in PTEN expression level lead to increased frequencies of certain tumors, particularly in breast $^{89,90}$. Conversely, beneficial effects are also reported. For example, VO-Ohpic inhibited cell viability, cell proliferation, and colony formation, and induced senescence in hepatocellular carcinoma cells ${ }^{91}$. We provide proof-ofprinciple evidence that pharmacological inhibition of PTEN is likely to be a promising approach in treating depression. In addition, our data indicate that CRS decreased the mouse body weight and inhibition of PTEN by VO-Ohpic reversed this change (Fig. 1h), raising a possibility that PTEN plays a critical role in leptin signaling pathway, regulating energy balance ${ }^{92}$.

\section{Acknowledgements}

This work is supported by the Shanghai Municipal Science and Technology Programs (19490714300), the Shanghai Key Project of Science and Technology (2018SHZDZX05), the Shanghai Pujiang Program (20PJ1413300), the National Natural Science Foundation of China (91232724, 81571332, 81930033, and 31671061), the National key R\&D program of China (2017YFA0104002), Collaborative Innovation Program of Shanghai Municipal Health Commission (2020CXJQ01), Shanghai Municipal Science and Technology Major Project (2018SHZDZX01), and ZJ Lab. 


\section{Author details}

${ }^{1}$ Key Laboratory of Arrhythmias, Ministry of Education of China, East Hospital, and Department of Anatomy and Neurobiology, Tongji University School of Medicine, Shanghai 200092, China. ${ }^{2}$ Department of Anatomy, Histology and Embryology, School of Basic Medical Sciences, Fudan University, Shanghai 200032, China. ${ }^{3}$ Shanghai Tenth People's Hospital, Clinical Medical School of

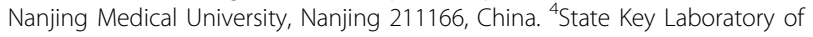
Medical Neurobiology and MOE Frontiers Center for Brain Science, Institutes of Brain Science, Fudan University, Shanghai 200032, China. ${ }^{5}$ Clinical Research Center and Division of Mood Disorders, Shanghai Mental Health Center, Shanghai Jiao Tong University School of Medicine, Shanghai 200030, China. ${ }^{6}$ CAS Center for Excellence in Brain Science and Intelligence Technology, Shanghai 200031, China. ${ }^{7}$ Department of Laboratory Animal Science, Fudan University, Shanghai 200032, China

\section{Conflict of interest}

The authors declare no competing interests.

\section{Publisher's note}

Springer Nature remains neutral with regard to jurisdictional claims in published maps and institutional affiliations.

Supplementary information The online version contains supplementary material available at https://doi.org/10.1038/s41398-021-01312-y.

Received: 28 September 2020 Revised: 24 February 2021 Accepted: 11 March 2021

Published online: 26 March 2021

\section{References}

1. Krishnan, V. \& Nestler, E. J. The molecular neurobiology of depression. Nature 455, 894-902 (2008).

2. Duman, R. S., Aghajanian, G. K., Sanacora, G. \& Krystal, J. H. Synaptic plasticity and depression: new insights from stress and rapid-acting antidepressants. Nat. Med. 22, 238-249 (2016).

3. Walker, E. R., McGee, R. E. \& Druss, B. G. Mortality in mental disorders and global disease burden implications: a systematic review and meta-analysis. JAMA Psychiatry 72, 334-341 (2015).

4. Network and Pathway Analysis Subgroup of Psychiatric Genomics Consortium Psychiatric genome-wide association study analyses implicate neuronal, immune and histone pathways. Nat. Neurosci. 18, 199-209 (2015).

5. Kaufman, J. et al. Social supports and serotonin transporter gene moderate depression in maltreated children. Proc. Natl Acad. Sci. USA 101, 17316-17321 (2004).

6. Kendler, K. S., Myers, J. \& Halberstadt, L. J. Should the diagnosis of major depression be made independent of or dependent upon the psychosocial context? Psychol. Med. 40, 771-780 (2010).

7. Levinson, D. F. et al. Genetic studies of major depressive disorder: why are there no genome-wide association study findings and what can we do about it? Biol. Psychiatry 76, 510-512 (2014).

8. Krishnan, $\mathrm{V}$. et al. Molecular adaptations underlying susceptibility and resistance to social defeat in brain reward regions. Cell 131, 391-404 (2007).

9. Berton, O. et al. Essential role of BDNF in the mesolimbic dopamine pathway in social defeat stress. Science 311, 864-868 (2006).

10. Berton, $\mathrm{O}$. et al. Induction of deltaFosB in the periaqueductal gray by stress promotes active coping responses. Neuron 55, 289-300 (2007).

11. Natarajan, R., Forrester, L., Chiaia, N. L. \& Yamamoto, B. K. Chronic-stressinduced behavioral changes associated with subregion-selective serotonin cell death in the dorsal raphe. J. Neurosci. 37, 6214-6223 (2017).

12. Liu, R. J. \& Aghajanian, G. K. Stress blunts serotonin- and hypocretin-evoked EPSCs in prefrontal cortex: role of corticosterone-mediated apical dendritic atrophy. Proc. Natl Acad. Sci. USA 105, 359-364 (2008).

13. Cai, L. et al. Epstein-Barr virus-encoded microRNA BART1 induces tumour metastasis by regulating PTEN-dependent pathways in nasopharyngeal carcinoma. Nat. Commun. 6, 7353 (2015).

14. Chen, M. et al. An aberrant SREBP-dependent lipogenic program promotes metastatic prostate cancer. Nat. Genet. 50, 206-218 (2018).
15. Lee, Y. R., Chen, M. \& Pandolfi, P. P. The functions and regulation of the PTEN tumour suppressor: new modes and prospects. Nat. Rev. Mol. Cell Biol. 19 547-562 (2018).

16. Backman, S., Stambolic, V. \& Mak, T. PTEN function in mammalian cell size regulation. Curr. Opin. Neurobiol. 12, 516-522 (2002).

17. Knafo, S. et al. PTEN recruitment controls synaptic and cognitive function in Alzheimer's models. Nat. Neurosci. 19, 443-453 (2016).

18. Liu, K., Tedeschi, A., Park, K. K. \& He, Z. Neuronal intrinsic mechanisms of axon regeneration. Annu. Rev. Neurosci. 34, 131-152 (2011).

19. Endersby, R. \& Baker, S. J. PTEN signaling in brain: neuropathology and tumorigenesis. Oncogene 27, 5416-5430 (2008).

20. Chen, C. J. et al. Therapeutic inhibition of $\mathrm{mTORC2}$ rescues the behavioral and neurophysiological abnormalities associated with Pten-deficiency. Nat. Med. 25, 1684-1690 (2019).

21. Borniger, J. C. et al. Behavioral abnormalities in mice lacking mesenchymespecific Pten. Behavioural Brain Res. 304, 80-85 (2016).

22. Hsiung, S. C. et al. Attenuated 5-HT1A receptor signaling in brains of suicide victims: involvement of adenylyl cyclase, phosphatidylinositol 3-kinase, Akt and mitogen-activated protein kinase. J. Neurochem. 87, 182-194 (2003).

23. Karege, F. et al. Alterations in phosphatidylinositol 3-kinase activity and PTEN phosphatase in the prefrontal cortex of depressed suicide victims. Neuropsychobiology 63, 224-231 (2011).

24. Zhang, X. C., Piccini, A., Myers, M. P., Van Aelst, L. \& Tonks, N. K. Functional analysis of the protein phosphatase activity of PTEN. Biochem. J. 444, 457-464 (2012).

25. Jaworski, J., Spangler, S., Seeburg, D. P., Hoogenraad, C. C. \& Sheng, M. Control of dendritic arborization by the phosphoinositide-3'-kinase-Akt-mammalian target of rapamycin pathway. J. Neurosci. 25, 11300-11312 (2005).

26. Manji, H. K., Drevets, W. C. \& Charney, D. S. The cellular neurobiology of depression. Nat. Med. 7, 541-547 (2001).

27. Bulteau, S. et al. Depression reappraisal and treatment effect: will response shift help improve the estimation of treatment efficacy in trials for mood disorders? Front. Psychiatry 10, 420 (2019).

28. Ni, Z. et al. A new pathway of glucocorticoid action for asthma treatment through the regulation of PTEN expression. Respir. Res. 12, 47 (2011).

29. Magarinos, A. M. \& McEwen, B. S. Stress-induced atrophy of apical dendrites of hippocampal CA3C neurons: involvement of glucocorticoid secretion and excitatory amino acid receptors. Neuroscience 69, 89-98 (1995).

30. Peritore, A. F. et al. The role of annexin A1 and formyl peptide receptor 2/ 3 signaling in chronic corticosterone-induced depression-like behaviors and Impairment in hippocampal-dependent memory. CNS Neurol. Disord. Drug Targets 19, 27-43 (2020).

31. Lesche, R. et al. Cre/loxP-mediated inactivation of the murine Pten tumor suppressor gene. Genesis 32, 148-149 (2002).

32. Gorski, J. A., Talley, T., Qiu, M., Puelles, L. \& Jones, K. R. Cortical excitatory neurons and glia, but not GABAergic neurons, are produced in the Emx1expressing lineage. J. Neurosci. 22, 6309-6314 (2002).

33. Cheng, Y. et al. Neurotrophic factor-alpha1 prevents stress-induced depression through enhancement of neurogenesis and is activated by rosiglitazone. Mol. Psychiatry 20, 744-754 (2015).

34. Zhao, D. et al. Pharmacologic activation of cholinergic alpha7 nicotinic receptors mitigates depressive-like behavior in a mouse model of chronic stress. J. Neuroinflammation 14, 234 (2017).

35. Mak, L. H., Vilar, R. \& Woscholski, R. Characterisation of the PTEN inhibitor VOOHpic. J. Chem. Biol. 3, 157-163 (2010).

36. Johnson, T. A. \& Singla, D. K. PTEN inhibitor VO-OHpic attenuates inflammatory M1 macrophages and cardiac remodeling in doxorubicin-induced cardiomyopathy. Am. J. Physiol. Heart Circ. Physiol. 315, H1236-H1249 (2018).

37. Yue, F. et al. PTEN inhibition ameliorates muscle degeneration and improves muscle function in a mouse model of Duchenne muscular dystrophy. Mol. Ther. 29, 132-148 (2021).

38. Chen, L., et al. Pten is a key intrinsic factor regulating raphe $5-H T$ neuronal plasticity and depressive behaviors in mice. Transl. Psychiatry (in press).

39. Song, N. N. et al. Reducing central serotonin in adulthood promotes hippocampal neurogenesis. Sci. Rep. 6, 20338 (2016).

40. Zhang, Q., Huang, Y., Zhang, L., Ding, Y. Q. \& Song, N. N. Loss of Satb2 in the cortex and hippocampus leads to abnormal behaviors in mice. Front Mol. Neurosci. 12, 33 (2019).

41. Dai, J. X. et al. Enhanced contextual fear memory in central serotonin-deficient mice. Proc. Natl Acad. Sci. USA 105, 11981-11986 (2008). 
42. Srinivasan, A., Srinivasan, A. \& Ferland, R. J. AutoSholl allows for automation of Sholl analysis independent of user tracing. J. Neurosci. Methods 331, 108529 (2020).

43. Ngoupaye, G. T., Yassi, F. B., Bahane, D. A. N. \& Bum, E. N. Combined corticosterone treatment and chronic restraint stress lead to depression associated with early cognitive deficits in mice. Metab. Brain Dis. 33, 421-431 (2018).

44. Ball, K. T. et al. Chronic restraint stress during withdrawal increases vulnerability to drug priming-induced cocaine seeking via a dopamine D1-like receptormediated mechanism. Drug Alcohol Depend. 187, 327-334 (2018).

45. Wang, S. et al. Agarwood essential oil ameliorates restrain stress-induced anxiety and depression by inhibiting HPA axis hyperactivity. Int. J. Mol. Sci. 19, 3468 (2018).

46. Agrawal, L. et al. Therapeutic potential of serotonin 4 receptor for chronic depression and its associated comorbidity in the gut. Neuropharmacology 166, 107969 (2020).

47. Borrow, A. P. et al. Chronic variable stress alters hypothalamic-pituitaryadrenal axis function in the female mouse. Physiol. Behav. 209, 112613 (2019).

48. Davidson, R. J., Pizzagalli, D., Nitschke, J. B. \& Putnam, K. Depression: perspectives from affective neuroscience. Annu Rev. Psychol. 53, 545-574 (2002).

49. Liu, W. et al. The role of neural plasticity in depression: from hippocampus to prefrontal cortex. Neural Plast. 2017, 6871089 (2017).

50. Alemu, J. L., Elberling, F., Azam, B., Pakkenberg, B. \& Olesen, M. V. Electroconvulsive treatment prevents chronic restraint stress-induced atrophy of the hippocampal formation-A stereological study. Brain Behav. 9, e01195 (2019).

51. Ramkumar, K. et al. Reversal of stress-induced dendritic atrophy in the prefrontal cortex by intracranial self-stimulation. J. Neural Transm. 119, 533-543 (2012).

52. Zanoveli, J. M. et al. Depression associated with diabetes: from pathophysiology to treatment. Curr. Diabetes Rev. 12, 165-178 (2016).

53. Heck, S. O., Zborowski, V. A., Pinton, S. \& Nogueira, C. W. Pro-apoptotic cell signaling in the prefrontal cortex contributes to depressive-/anxiogenic-like behavioral phenotype of mice subchronically exposed to dexamethasone. J. Chem. Neuroanat. 100, 101663 (2019).

54. Mesripour, A., Alhimma, F. \& Hajhashemi, V. The effect of vitamin B6 on dexamethasone-induced depression in mice model of despair. Nutritional Neurosci. 22, 744-749 (2019).

55. Lamontagne, S. J., Melendez, S. I. \& Olmstead, M. C. Investigating dopamine and glucocorticoid systems as underlying mechanisms of anhedonia. Psychopharmacology 235, 3103-3113 (2018).

56. Madalena, K. M. \& Lerch, J. K. The effect of glucocorticoid and glucocorticoid receptor interactions on brain, spinal cord, and glial cell plasticity. Neural Plasticity 2017, 8640970 (2017).

57. Commons, K. G., Cholanians, A. B., Babb, J. A. \& Ehlinger, D. G. The rodent forced swim test measures stress-coping strategy, not depression-like behavior. ACS Chem. Neurosci. 8, 955-960 (2017).

58. Baumgarten, H.G. [Anatomy and physiology of the central serotonin system]. Arzneimittel-Forschung Suppl. 27, 23-25 (1981). in German.

59. Leon-Dominguez, U. \& Leon-Carrion, J. Prefrontal neural dynamics in consciousness. Neuropsychologia 131, 25-41 (2019).

60. van Bodegom, M., Homberg, J. R. \& Henckens, M. Modulation of the hypothalamic-pituitary-adrenal axis by early life stress exposure. Front Cell Neurosci. 11, 87 (2017)

61. Preil, J. et al. Regulation of the hypothalamic-pituitary-adrenocortical system in mice deficient for $\mathrm{CRH}$ receptors 1 and 2. Endocrinology 142, 4946-4955 (2001).

62. Schreiber, A. L., Lu, Y. L., Baynes, B. B., Richardson, H. N. \& Gilpin, N. W. Corticotropin-releasing factor in ventromedial prefrontal cortex mediates avoidance of a traumatic stress-paired context. Neuropharmacology $\mathbf{1 1 3}$ 323-330 (2017).

63. Barfield, E. T. \& Gourley, S. L. Prefrontal cortical trkB, glucocorticoids, and their interactions in stress and developmental contexts. Neurosci. Biobehav. Rev. 95, 535-558 (2018)

64. Swaab, D. F., Bao, A. M. \& Lucassen, P. J. The stress system in the human brain in depression and neurodegeneration. Ageing Res. Rev. 4, 141-194 (2005).

65. Butler, M. G. et al. Subset of individuals with autism spectrum disorders and extreme macrocephaly associated with germline PTEN tumour suppressor gene mutations. J. Med. Genet. 42, 318-321 (2005).
66. Kwon, C. H. et al. Pten regulates neuronal arborization and social interaction in mice. Neuron 50, 377-388 (2006).

67. Tilot, A. K. et al. Germline disruption of Pten localization causes enhanced sexdependent social motivation and increased glial production. Hum. Mol. Genet. 23, 3212-3227 (2014)

68. Frazier, T.W. Autism spectrum disorder associated with germline heterozygous PTEN mutations.Cold Spring Harb. Perspect. Med. 9, a037002 (2019).

69. Jurado, S. et al. PTEN is recruited to the postsynaptic terminal for NMDA receptor-dependent long-term depression. EMBO J. 29, 2827-2840 (2010).

70. Weston, M. C., Chen, H. \& Swann, J. W. Multiple roles for mammalian target of rapamycin signaling in both glutamatergic and GABAergic synaptic transmission. J. Neurosci. 32, 11441-11452 (2012).

71. Liu, R. J. et al. GSK-3 inhibition potentiates the synaptogenic and antidepressant-like effects of subthreshold doses of ketamine. Neuropsychopharmacology 38, 2268-2277 (2013).

72. Voleti, B. et al. Scopolamine rapidly increases mammalian target of rapamycin complex 1 signaling, synaptogenesis, and antidepressant behavioral responses. Biol. Psychiatry 74, 742-749 (2013).

73. Li, N. et al. mTOR-dependent synapse formation underlies the rapid antidepressant effects of NMDA antagonists. Science 329, 959-964 (2010).

74. Amidfar, M. et al. The role of NMDA receptor in neurobiology and treatment of major depressive disorder: evidence from translational research. Prog. Neuro-Psychopharmacol. Biol. Psychiatry 94, 109668 (2019).

75. Duman, R. S. Neurobiology of stress, depression, and rapid acting antidepressants: remodeling synaptic connections. Depression Anxiety 31, 291-296 (2014).

76. Boosani, C. S. \& Agrawal, D. K. PTEN modulators: a patent review. Expert Opin. Therapeutic Pat. 23, 569-580 (2013).

77. Sanchez-Puelles, C. et al. PTEN activity defines an axis for plasticity at corticoamygdala synapses and influences social behavior. Cereb. Cortex 30, 505-524 (2020).

78. Takeuchi, K. et al. Dysregulation of synaptic plasticity precedes appearance of morphological defects in a Pten conditional knockout mouse model of autism. Proc. Natl Acad. Sci. USA 110, 4738-4743 (2013).

79. Skelton, P. D., Frazel, P. W., Lee, D., Suh, H. \& Luikart, B. W. Pten loss results in inappropriate excitatory connectivity. Mol. Psychiatry 24, 1627-1640 (2019).

80. Santos, V. R. et al. PTEN deletion increases hippocampal granule cell excitability in male and female mice. Neurobiol. Dis. 108, 339-351 (2017).

81. Bessa, J. M. et al. The mood-improving actions of antidepressants do not depend on neurogenesis but are associated with neuronal remodeling. Mol. Psychiatry 14, 764-773. 739 (2009).

82. Sano, T. et al. Differential expression of MMAC/PTEN in glioblastoma multiforme: relationship to localization and prognosis. Cancer Res. 59, 1820-1824 (1999).

83. Shen, W. H. et al. Essential role for nuclear PTEN in maintaining chromosomal integrity. Cell 128, 157-170 (2007).

84. Chen, Z. H. et al. PTEN interacts with histone $\mathrm{H} 1$ and controls chromatin condensation. Cell Rep. 8, 2003-2014 (2014).

85. Gong, L. et al. Nuclear PTEN tumor-suppressor functions through maintaining heterochromatin structure. Cell Cycle 14, 2323-2332 (2015).

86. Spinelli, L., Lindsay, Y. E. \& Leslie, N. R. PTEN inhibitors: an evaluation of current compounds. Adv. Biol. Regul. 57, 102-111 (2015).

87. Zhu, Y. B., Ding, N., Yi, H. L. \& Li, Z. Q. The expression of overexpressed PTEN enhanced IR-induced apoptosis of myocardial cells. Eur. Rev. Med. Pharmacol. Sci. 23, 4406-4413 (2019).

88. Li, J. et al. A novel pharmacological strategy by PTEN inhibition for improving metabolic resuscitation and survival after mouse cardiac arrest. Am. J. Physiol. Heart Circulatory Physiol. 308, H1414-H1422 (2015).

89. Alimonti, A. et al. Subtle variations in Pten dose determine cancer susceptibility. Nat. Genet. 42, 454-458 (2010).

90. Trotman, L. C. et al. Pten dose dictates cancer progression in the prostate. PLoS Biol. 1, E59 (2003).

91. Augello, G. et al. A PTEN inhibitor displays preclinical activity against hepatocarcinoma cells. Cell Cycle 15, 573-583 (2016).

92. Ning, K. et al. A novel leptin signalling pathway via PTEN inhibition in hypothalamic cell lines and pancreatic beta-cells. EMBO J. 25, 2377-2387 (2006). 